\title{
Experimental characterization of the dynamics of IDPs and IDRs by NMR
}

Nicolas Bolik-Coulon, Guillaume Bouvignies, ${ }^{*}$ Ludovic Carlier, Fabien Ferrage*

Laboratoire des biomolécules, LBM, Département de chimie, École normale supérieure, PSL University, Sorbonne Université, CNRS, 75005 Paris, France

Fabien.Ferrage@ens.fr

Guillaume.Bouvignies@ens.fr

\section{Abstract:}

Nuclear Magnetic Resonance is a unique approach to obtain at the same time information on dynamics over a broad range of timescales and high-resolution information in disordered proteins. In this chapter, we will review the different methods available to characterize motions in disordered proteins. We first focus on dynamics in the picosecond-nanosecond range, which is characterized by nuclear spin relaxation measurements. We will focus then on slower processes, mostly in the millisecond range, studied by chemical-exchange NMR, discussing in particular the dynamics of the binding of disordered proteins to their targets and their dynamics in these complexes. 


\section{Table of Contents}

1. Adapting the toolbox: comparison of NMR investigations of folded and disordered proteins

2. Determining motions in IDRs and IDPs in the picosecond-nanosecond range

2.1. Nuclear spin relaxation to probe ps-ns motions ............................................... 4

2.2. Measurement of longitudinal auto- and cross-relaxation .................................... 6

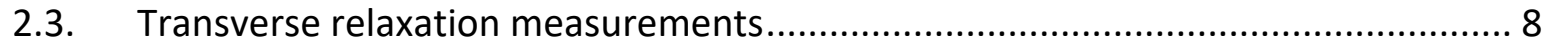

2.4. Cross-correlated relaxation measurements ...................................................... 9

2.5. Models to interpret ps-ns motions in IDRs and IDPs ........................................ 10

2.6. Comparison with molecular dynamics simulations ............................................ 13

3. Microsecond-millisecond motions in IDPs and IDRs: binding ................................... 14

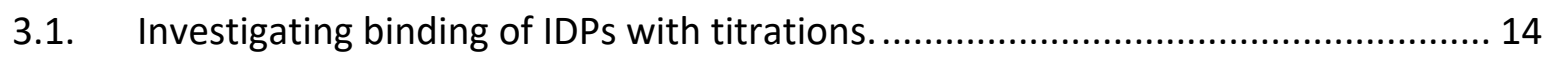

3.1.1. Atomic resolution of the interaction and model of binding ......................... 15

3.1.2. Extracting thermodynamics and kinetics information from NMR titration

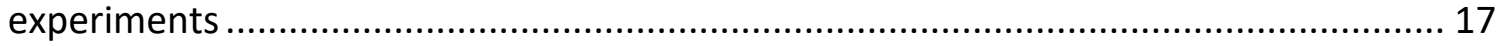

3.2. Investigations of binding mechanisms with chemical-exchange NMR .................. 18

3.3. Other methods to characterize ps-ns dynamics in complexes of IDPs and IDRs

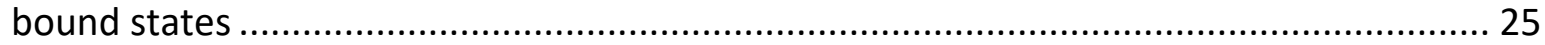

3.4. Amide proton exchange: from false positives to insightful information ............... 26

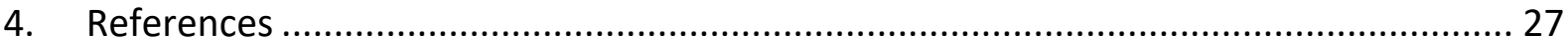


1. Adapting the toolbox: comparison of NMR investigations of folded and disordered proteins

Nuclear magnetic resonance has proven to be a unique tool to study the dynamics of biological macromolecules at atomic resolution. The entire protein NMR toolbox has been developed over about three decades for applications on folded proteins. We can consider that a folded protein is defined by a representative conformation, while additional conformational states can be represented as perturbations to this representative structure. On the other hand, intrinsically disordered proteins (IDPs) and regions (IDRs) have emerged over the last two decades as proteins with an extremely broad conformational space and detectable structural features are a limited restriction of their conformational space. With little doubt, the dynamics of proteins, defined by the mechanistic paths of exploration of the conformational space and the timescales of this exploration, differ drastically between folded and disordered proteins.

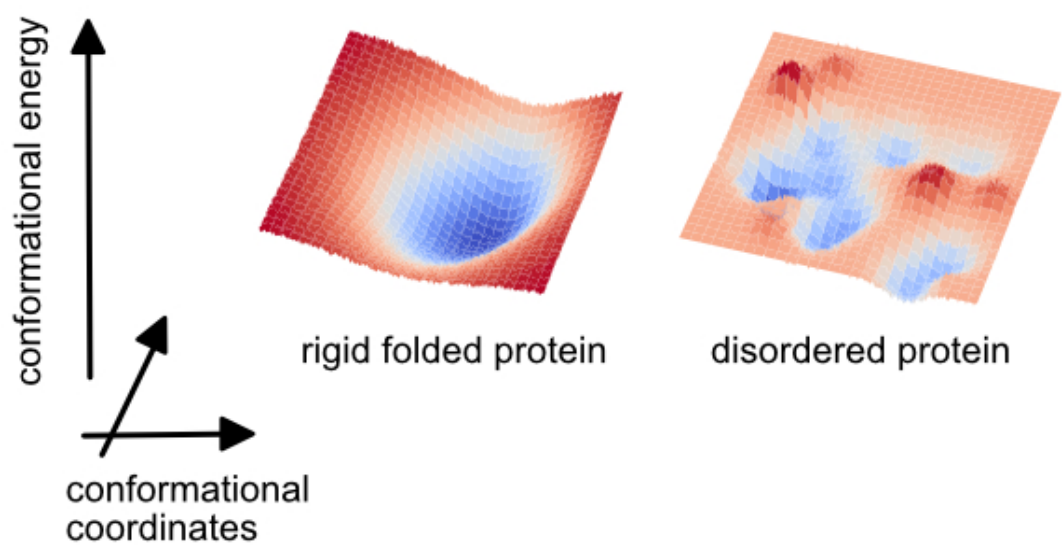

Figure 1. Schematic representation of the conformational energy profiles of a rigid folded protein and an intrinsically disordered protein.

As can be seen in Figure 1, many more conformational states can be populated in disordered proteins and there are many more mechanistic pathways between these conformational states. A first clear consequence is that the models of motions routinely used to interpret NMR experiments in folded proteins are often ill-suited to describe dynamics in disordered proteins. For instance, the measurement and analysis of NMR relaxation rates provides direct information on motions in proteins on picosecond to nanosecond timescales. Yet, with the exception of short disordered regions in mostly folded proteins, it is generally impossible to define an overall rotational diffusion tensor and distinguish overall and internal motions in IDPs. Another clear difference between folded and disordered proteins relates to slower 
motions on micro- to second timescales. The conformational energy surface of folded proteins can often be represented to the first order by a single and relatively deep minimum. Very frequently, additional conformational states can be accessed if they are not too far off in energy: hence many methods, based on the exploitation of chemical exchange, have been developed over the years to characterize such excited states of proteins, as well as the kinetics and thermodynamics of their exploration. Interestingly, the exploration of the main conformational space of IDPs is generally faster than the microsecond and slower timescales that give rise to chemical exchange. In the presence of many states accessible with such low barriers and similar energies, these systems are associated with a very large partition function. Thus, excited states in IDPs, with an energy higher by a few $\mathrm{kT}$, are expected to have a relative population at a low level, well below detection limits. Thus, chemical- exchange methods have not been successful at exploring intrinsic excited states in IDPs. On the other hand, these methods are of utmost importance to study engineered excited states of proteins: the state of a disordered protein bound to a partner (often a folded domain), in exchange with a dominant free state.

Another important difference of disordered proteins, as compared to folded proteins, is the constant solvent accessibility of all sites in the protein. In particular, labile amide protons from the backbone in IDPs are accessible to the solvent and hydrogen bonded to water molecules. Thus, amide proton exchange is much more prevalent in IDPs than in folded proteins. Although this is an insightful property, this exchange is often degrading the accuracy and precision of experiments that were designed for folded proteins with limited exchange of amide protons with the solvent.

\section{Determining motions in IDRs and IDPs in the picosecond-nanosecond range}

\subsection{Nuclear spin relaxation to probe ps-ns motions}

The irreversible evolution of the spin system towards equilibrium originates in the fluctuation of interactions at the molecular scale. Thus, relaxation rates open a rich window on molecular motions through the measurement of macroscopic properties. Three main interactions lead to spin relaxation in proteins: the dipole-dipole (DD) interactions between two spins (nucleus and/or electron), the Chemical Shift Anisotropy (CSA) and the quadrupolar interaction ${ }^{1}$. With few exceptions ${ }^{2}$ relaxation studies of IDPs and IDRs have been performed with spin $1 / 2$ nuclei 
so that only the DD and CSA relaxation mechanisms need to be considered. The theory of nuclear spin relaxation by these interactions is well understood and has been presented extensively in a number of textbooks and reviews ${ }^{3-5}$.

The fluctuations of nuclear spin interactions are described by correlation functions: either with an auto-correlation function (an interaction with itself) or a cross-correlation function (the correlation of an interaction with another interaction). The molecular origin of fluctuations of internal interactions in disordered proteins are reorientation motions: local librations, dihedral angle jumps between rotamers, rotational diffusion of segments of the polypeptide chain. The correlation function will typically decay to zero within a few nanoseconds or a few tens of nanoseconds. Thus, relaxation can be used to probe motions that occur between low picoseconds and low tens of nanoseconds.

The Fourier transform of the correlation function is the spectral density function. Relaxation rates are expressed as linear combination of the spectral density function taken at eigenfrequencies of the spin system. For example, the nitrogen-15 transverse relaxation rate in an isolated ${ }^{15} \mathrm{~N}-{ }^{1} \mathrm{H}$ pair, $R_{2}$, depends on the spectral density function evaluated at the following frequencies: $0, \omega_{H}, \omega_{N}, \omega_{H}-\omega_{N}$ and $\omega_{H}+\omega_{N}$ :

$$
\begin{aligned}
R_{2}=\frac{d_{N H}}{8}(4 J(0) & \left.+J\left(\omega_{H}-\omega_{N}\right)+3 J\left(\omega_{N}\right)+6 J\left(\omega_{H}\right)+6 J\left(\omega_{H}+\omega_{N}\right)\right) \\
+ & \frac{c_{N}^{2}}{6}\left(4 J(0)+3 J\left(\omega_{N}\right)\right)
\end{aligned}
$$

with $J(\omega)$ the spectral density function, $\omega_{i}=\gamma_{i} B_{0}$ the Larmor frequency at magnetic field $\mathrm{B}_{0}$ of nucleus $i$ of gyromagnetic ratio $\gamma_{i}, \mathrm{~d}_{\mathrm{NH}}$ the dipolar coefficient for the ${ }^{15} \mathrm{~N}-{ }^{1} \mathrm{H}$ spin pair $d_{N H}=$ $\frac{\mu_{0} \hbar^{2} \gamma_{H}^{2} \gamma_{N}^{2}}{4 \pi r_{N H}^{6}}, \mu_{0}$ the permeability of vacuum, $\hbar$ the Plank constant divided by $2 \pi$ and $r_{N H}$ the internuclear distance between the nitrogen and proton nuclei, and $c_{N}=\frac{\Delta \sigma_{N} \omega_{N}}{\sqrt{3}}$ the nitrogen CSA constant with $\Delta \sigma_{N}=\sigma_{N, \|}-\sigma_{N, \perp}$ defined using the longitudinal and orthogonal CSA tensor components.

Overall, for $a^{15} \mathrm{~N}-{ }^{1} \mathrm{H}$ pair on a protein, the measurement of sets of relaxation rates allows the sampling of the spectral density function in 0 and at frequencies ranging from $40 \mathrm{MHz}$ to 1.1 $\mathrm{GHz}$ (corresponding to magnetic field from $400 \mathrm{MHz}$ to $1 \mathrm{GHz}$ ), corresponding to motions on the ps-ns timescale, which corresponds in large part to the decay timescales of the correlation function. 
Quantitative descriptions of dynamics require the definition of a model of motion, i.e. an expression for the correlation function. In its most simple form, the correlation function only accounts for the isotropic reorientation of a molecule in solution, with no internal degree of freedom, a process often referred to as global tumbling and takes the form:

$$
C(t)=\frac{1}{5} e^{-t / \tau_{c}}
$$

where $\tau_{c}$ is the global tumbling correlation time, on the order of few nanoseconds for small molecules to few hundreds for large proteins. The Fourier transform of this correlation function leads to the following form of the spectral density function:

$$
J(\omega)=\frac{2}{5} \frac{\tau_{c}}{\left(1+\omega^{2} \tau_{c}^{2}\right)}
$$

Motions in proteins can rarely be described with a single correlation time. In IDPs and IDRs, several reorientation motions are superimposed and lead to multiexponential decays of correlation functions. The measurement and analysis of large sets of relaxation rates allow for the description of motions over two to three orders of magnitude of correlation times as will be illustrated in the following sections.

Ample motions around 1 ns (very fast for a protein) lead to small values of the correlation function $\mathrm{J}(\mathrm{w})$ over the entire range of frequencies probed by relaxation in IDPs and IDRs. In particular, the spectral density J(0), which typically dominates the contributions to transverse relaxation, is particularly small. The resulting low relaxation rates allow for a significant amount of polarization to be preserved in long experiments, opening the way to the development of complex experiments in IDPs and IDRs ${ }^{6}$. In the case of IDPs and IDRs, specific pulse sequences have been developed to improve the resolution and efficiency of relaxation measurements 7,8

\subsection{Measurement of longitudinal auto- and cross-relaxation}

Steady-state ${ }^{15} \mathrm{~N}-\left\{{ }^{1} \mathrm{H}\right\}$ nuclear Overhauser effects (NOEs) are often employed as a single relaxation-based experimental evidence of the disordered nature of a protein region. The method consists in measuring the nitrogen-15 polarization at the steady-state under the effective saturation of protons ${ }^{9}$. Methods to measure NOEs have been recently improved to reduce artefacts ${ }^{10,11}$. A specificity of IDPs is the presence of exchange of labile amide protons 
with the solvent. Steady-state NOEs are remarkably resilient to this effect, as long as the frequency of saturation of the protons is significantly higher than the kinetic constant of exchange.
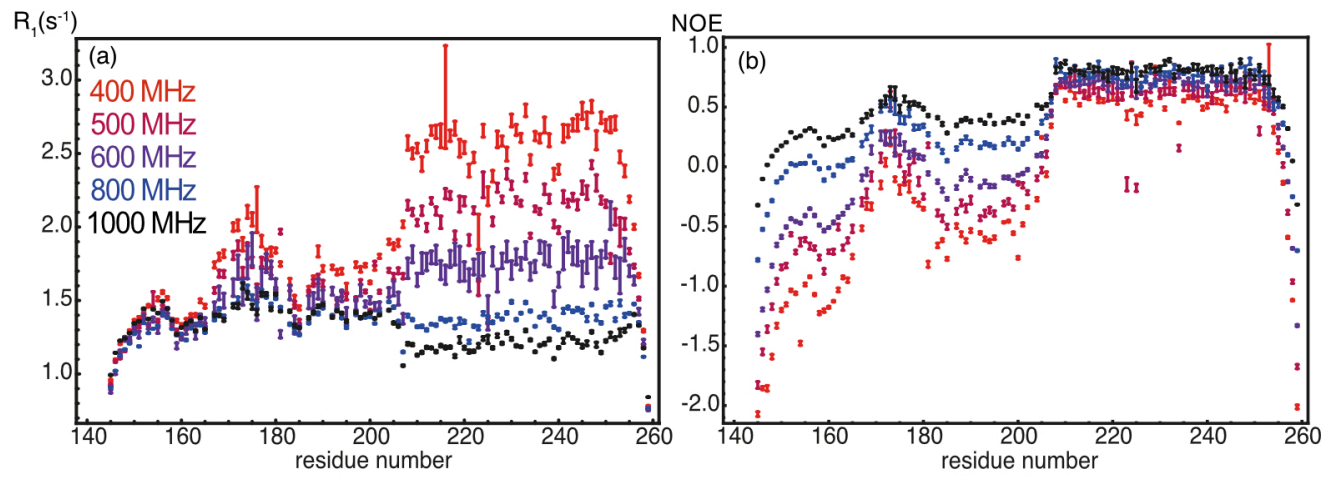

Figure 2. (a) Nitrogen-15 longitudinal relaxation rates and (b) steady-state ${ }^{15} \mathrm{~N}-\left\{{ }^{1} \mathrm{H}\right\}$ nuclear Overhauser effects recorded on the protein Engrailed 2 at magnetic fields ranging between $400 \mathrm{MHz}(9.4 \mathrm{~T})$ and $1 \mathrm{GHz}(23.5 \mathrm{~T})$. This construction includes the homeodomain (residues 205-259), which comprises three $\alpha$-helices and a disordered $N$-terminal extension that features a hydrophobic cluster around residue 175. Adapted from ${ }^{12}$.

Steady-state ${ }^{15} \mathrm{~N}-\left\{{ }^{1} \mathrm{H}\right\}$ NOEs arise from cross-relaxation between proton and nitrogen-15 longitudinal polarizations. This cross-relaxation rate $\sigma_{\mathrm{NH}}$ is proportional to the spectral density function near the Larmor frequency of the proton, to a good approximation: $\sigma_{N H} \propto$ $J\left(0.87 \omega_{H}\right)$, where $\omega_{H}$ is the Larmor frequency of the proton ${ }^{13}$. Note that steady-state NOEs estimate the ratio between this cross-relaxation rate and the longitudinal relaxation rate of the nitrogen-15 nucleus. One measures the following ratio of intensities:

$$
N O E=\frac{I^{S S}}{I^{e q}}=1+\frac{\gamma_{H}}{\gamma_{N}} \frac{\sigma_{N H}}{R_{1}}
$$

where $I^{s s}$ is the intensity measured at the steady-state under proton saturation, and $l^{e q}$ the intensity measured at equilibrium, with no perturbation of the spin system. $\gamma_{N, H}$ are the gyromagnetic ratios of the nitrogen-15 and hydrogen nuclei, $R_{1}$ is the longitudinal relaxation rate of the nitrogen-15 nucleus. Most often, the longitudinal relaxation rate of nitrogen-15 is dominated by the spectral density at the Larmor frequency of the nitrogen-15 nucleus, $J\left(\omega_{N}\right)$. Thus, NOEs give a measure of the ratio $J\left(0.87 \omega_{H}\right) / J\left(\omega_{N}\right)$. As can be seen in Figure 2 , in the case of the protein Engrailed-2, which comprises an N-terminal disordered region followed by a folded homeodomain, NOEs are strongly field-dependent while $R_{1}$ are weakly fielddependent in the IDR ${ }^{12}$. Indeed, the spectral density at high frequency $J\left(0.87 \omega_{H}\right)$ varies 
significantly in the range of high magnetic fields currently accessible, while the spectral density $J\left(\omega_{N}\right)$ varies much less with the field. By contrast, steady-state NOEs vary weakly with the field in the folded domain, due to similar variations of the spectral densities $J\left(0.87 \omega_{H}\right)$ and $J\left(\omega_{N}\right)$. An important lesson of this study is that steady-state ${ }^{15} \mathrm{~N}-\left\{{ }^{1} \mathrm{H}\right\}$ NOEs are strongly fielddependent in IDPs. A ratio equal to 0.3 at a moderate field (e.g. $500 \mathrm{MHz}$ ) highlights the presence of slow tumbling structured elements. The same ratio of 0.3 at $1 \mathrm{GHz}$ corresponds to a fully disordered region. Hence, one should always indicate the magnetic field at which steady-state ${ }^{15} \mathrm{~N}-\left\{{ }^{1} \mathrm{H}\right\}$ NOEs are recorded, this is an important piece of information.

Longitudinal relaxation rates $R_{1}$ are necessary for the analysis of pico- to nanosecond motions in disordered proteins. Yet, as can be seen from Figure 2.a, nitrogen-15 longitudinal relaxation rates are not those with the most striking variations in IDPs. The main reason is that longitudinal relaxation rates depend on linear combinations of the spectral density function at the Larmor frequency of nitrogen-15 and close to the proton Larmor frequency. As we will see below, motions with correlation times around $\tau_{c}=1 \mathrm{~ns}$ dominate the reorientation dynamics of the backbone in IDPs. The reciprocal frequency is $\omega=10^{9} \mathrm{rad} . \mathrm{s}^{-1}$ or $\omega / 2 \pi=160$ $\mathrm{MHz}$. This frequency seats just in between the range of Larmor frequencies for nitrogen-15 and the proton for all high magnetic fields currently accessible. Hence, small variations of dynamics from site to site will lead to an increase (resp. decrease) of the spectral density $J\left(\omega_{N}\right)$, compensated by a decrease (resp. increase) of the spectral densities $J\left(\omega_{N}+\omega_{H}\right)$ and

$J\left(\omega_{N}-\omega_{H}\right)$, reducing variations of the longitudinal relaxation rate $R_{1}$. Note that the exact same trends enhance the site-to-site variations of the NOE ratio.

\subsection{Transverse relaxation measurements}

Transverse relaxation measurements usually open a rich window on the dynamics of proteins over many timescales: the rates are dominated by the spectral density function at zero frequency, $J(0)$, which is proportional to a weighted average of the correlation times that define reorientation motions in the pico- to nanosecond range. In addition, contributions due to chemical exchange (see below) lead to higher transverse relaxation rates in the presence of micro- and millisecond dynamics. Unfortunately, transverse nitrogen-15 relaxation rates in disordered proteins are quite difficult to evaluate accurately. The main reason is the sensitivity of the rates measured to the exchange of labile amide protons with the solvent. This process 
leads to apparent increase of transverse relaxation rates due to the chemical exchange between hydrogen-bound and deuterium bound nitrogen-15 ${ }^{14}$. In addition, the starting polarization of amide proton is strongly dependent on the partial saturation of water protons, which is relaxation-delay dependent. In addition, nitrogen-15 transverse relaxation times in IDPs are usually much longer than the maximum duration of the relaxation delay that is permitted on most high-resolution probes. Thus, only the beginning of the relaxation decay can be sampled, reducing the accuracy of the measurements. Several schemes have been proposed to improve the precision and accuracy of transverse relaxation rate measurements. In particular, the proton-decoupled Carr-Purcell-Meiboom-Gill sequence, introduced by Yuwen and Skrynnikov ${ }^{15}$ is an efficient alternative to conventional sequences developed for folded proteins.

\subsection{Cross-correlated relaxation measurements}

Relaxation may occur from the interference of two different relaxation mechanisms. Such cross-correlated relaxation rates often depend solely on the spectral density at zero frequency $J(0)$. Importantly, these rates are very weakly dependent on the presence of chemical exchange. They are thus an interesting alternative to tedious measurements of transverse relaxation rates. In particular, cross-relaxation rates $\eta_{x y}$ due to the cross-correlation of the nitrogen-15 chemical shift anisotropy (CSA) and the dipole-dipole (DD) coupling with the amide proton are insightful. The experiment developed to measure this rate is very robust ${ }^{16}$ and has been applied to several IDPs ${ }^{12,17}$. The only drawback of this experiment is the necessary measurement of relaxation to and from a nitrogen-15 polarization antiphase with the amide proton. Amide exchange with the solvent leads to a fast decay of this polarization, altering the precision of measurements, particularly if carried out at high temperature and/or high pH (higher than 6.5). In the case of Engrailed-2 homeoprotein (Figure 3), sharp variations of $\eta_{x y}$ transverse cross-relaxation rates were observed at all fields in both the ordered and disordered regions. As these variations were strongly correlated with the secondary structure propensities (SSP) of the protein, $\eta_{x y}$ were considered as the most relevant relaxation rates for characterizing order in IDPs and IDRs ${ }^{12}$. 

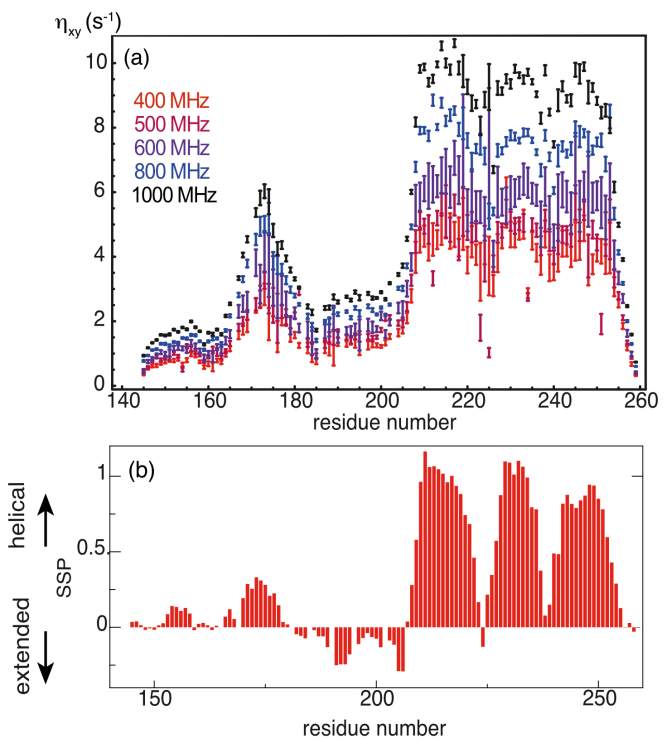

Figure 3. (a) CSA/DD cross-correlated cross-relaxation rates recorded on the protein Engrailed-2 at magnetic fields ranging between $400 \mathrm{MHz}(9.4 \mathrm{~T})$ and $1 \mathrm{GHz}$ (23.5 T). (b) Secondary structure propensities ${ }^{18}$ calculated from carbon-13 chemical shifts. This construction includes the homeodomain (residues 205-259), which comprises three $\alpha$ helices and a disordered $\mathrm{N}$-terminal extension that features a hydrophobic cluster around residue 175. Adapted from ${ }^{12}$.

Other cross-correlated relaxation rates can be measured in principle in disordered proteins. For instance $\mathrm{DD} / \mathrm{DD}$ cross-correlated relaxation measurements have been carried out to sample the dynamics of methylene side chain groups in an unfolded protein ${ }^{19}$. However, cross-correlated relaxation rates depend on the dynamics as well as the relative orientation of the two interactions. Note that the examples discussed above describe interactions with a mostly rigid relative orientation $\left(C S A\right.$ and $D D$ in ${ }^{15} \mathrm{~N}-{ }^{1} \mathrm{H}$ pairs or the two ${ }^{13} \mathrm{C}-{ }^{1} \mathrm{H}$ DD interactions in a methylene group). The broad conformational space of IDPs makes the wide variety of cross-correlated relaxation methods developed for folded proteins difficult to interpret in disordered proteins as the effects of average relative orientations (structure) and correlation times (dynamics) are difficult to deconvolute.

\subsection{Models to interpret ps-ns motions in IDRs and IDPs}

As mentioned in the introduction to this chapter, most models at hand to interpret nitrogen15 relaxation in intrinsically disordered proteins were developed and tested in folded proteins and are poorly adapted. In particular, the widely used model-free approach ${ }^{20,21}$ relies on a separation of two types of motions: a global motion, which is the rotational diffusion of the entire molecule, and internal motions. In IDPs, the lifetime of the overall conformation of the 
protein is comparable to its typical overall diffusion correlation time, defying the definition of an overall diffusion tensor. In the absence of a well-defined overall motion, a series of alternate approaches has been developed.

A simple way to analyze relaxation rates is to retrieve the values of the spectral density function from relaxation rates without trying to extract correlation times. The first step is quantitative ${ }^{13,22,23}$ and the exploitation of the spectral density values is rather qualitative ${ }^{24}$. A related approach consists in determining a distribution of correlation times from an ensemble of relaxation rates. This ill-posed problem is best approached with regularization methods and provides quantitative distributions of relaxation rates with few assumptions ${ }^{12}$. A good example is the Interpretation of Motions by a Projection onto an Array of Correlation Times (IMPACT). The range of correlation times characterized is defined by the range of eigenfrequencies covered in the relaxation dataset, which is defined by the types of nuclei and the range of magnetic fields. Then, an array of correlation times, equally spaced on a logarithmic scale is globally optimized. Finally, a discrete distribution of correlation times is obtained for each residue in the protein. In the protein Engrailed-2, motions in the disordered concentrate around $1 \mathrm{~ns}$, with significant contribution of slower motions in the hydrophobic core around residue 175 and broader distributions of sub-ns motions at both the $\mathrm{N}$-terminus and the C-terminus (Fig.4 ). By contrast, relaxation mostly characterizes overall rotational diffusion in the folded homeodomain (residues 205-259).

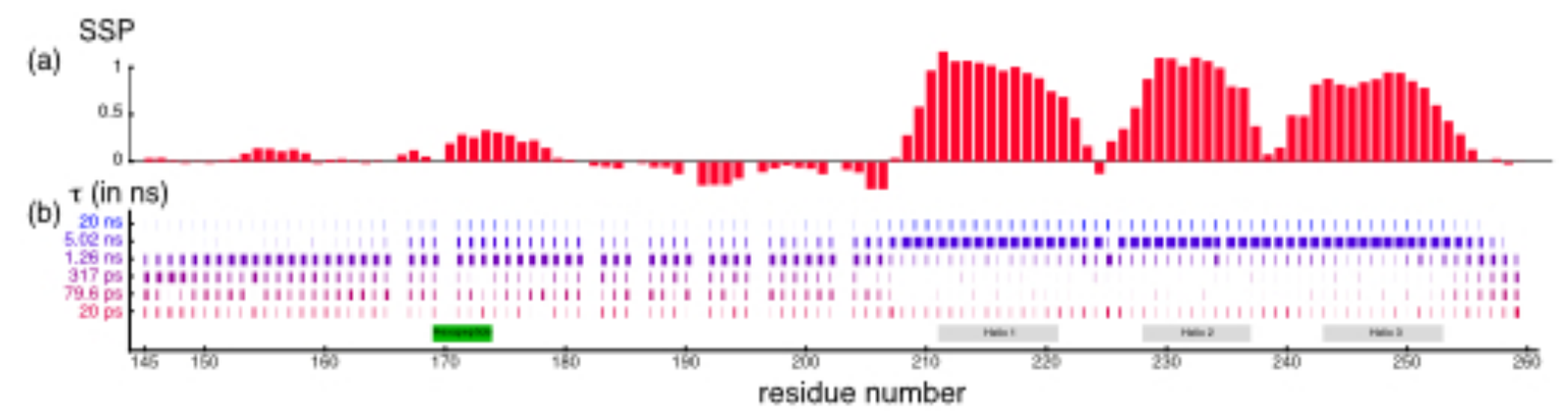

Figure 4. Dynamic characterization of Engrailed-2, a partially disordered protein by the IMPACT approach. (a) Secondary structure propensity of Engrailed-2. (b) Residue-specific distribution of correlation times. This graph should be read as vertical histograms representing the relative weight of each correlation time in the distribution. The height of each bar in the histogram Is also reflected in the color-coding for enhanced visibility. Reproduced with permission from ${ }^{12}$. 
Another efficient approach for the analysis of relaxation rates in disordered proteins is the use of a series of correlation times for motions covering different orders of magnitude of timescales ${ }^{17,25}$. Although the individual correlation times may only be effective correlation times and may not represent a single motion ${ }^{20,26}$, a careful analysis can be extremely insightful, identifying clearly different classes of motions (Fig. 5). In particular, a study at multiple magnetic fields over a broad range of temperatures has shown that three effective modes could be defined and analyzed together ${ }^{17}$ :

- Fast motions, likely librations of the hydrated peptide plans, with correlation times close to 50 ps and weakly temperature dependent.

- Intermediate motions, due to jumps of backbone dihedral angles leading to an effective correlation time close to $1 \mathrm{~ns}$.

- Slower motions with correlation times in the 3-10 ns range, corresponding to the rotational diffusion of segments of the peptide chain.

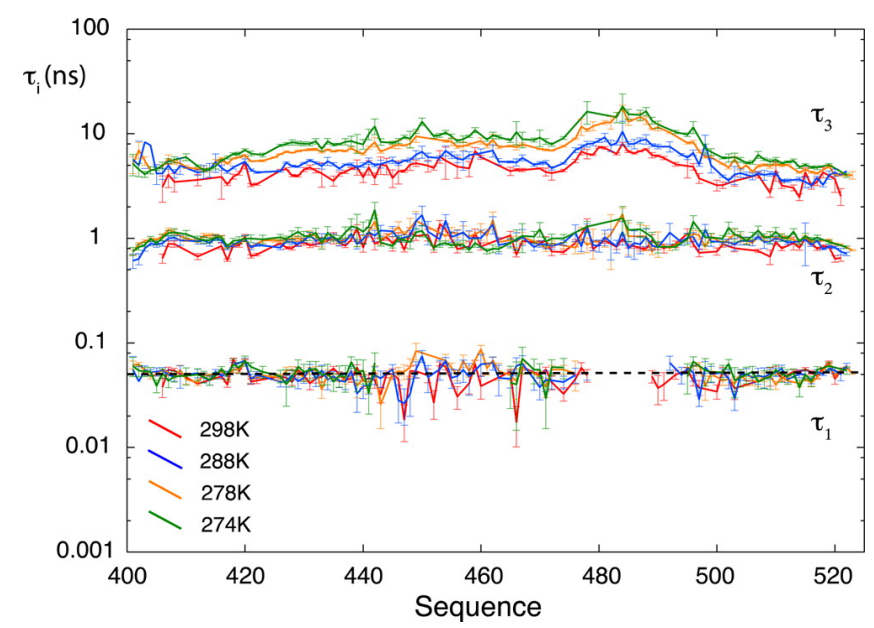

Figure 5. Analysis of a broad set of relaxation rates in the disordered protein N-Tail, using three discrete correlation times. Longitudinal and transverse relaxation rates were measured alongside transverse CSA/DD cross-correlated cross-relaxation rates and steady-state ${ }^{15} \mathrm{~N}-\left\{{ }^{1} \mathrm{H}\right\}$ nuclear Overhauser effects at four temperatures and four magnetic fields. Motions covering more than two orders of magnitude of correlation times can be analyzed from nitrogen-15 relaxation. Adapted with permission from ${ }^{17}$. Copyright 2016 American Chemical Society.

As disordered proteins explore a broad set of conformations, it is legitimate to hypothesize that its motions cover a continuous distribution of correlation times. Early studies by Buevich and Baum ${ }^{27,28}$ suggested the use of the Cole-Cole distribution ${ }^{29}$. Unfortunately, this empirical distribution, introduced to describe dielectric dispersion and absorption provides unphysical parameters when NMR relaxation is considered, as the spectral density function $J(\omega)$ diverges 
as $\omega \rightarrow 0$. More recently, Palmer and co-workers introduced another type of distribution, inverse Gaussian distributions, which are based on the principle of maximum entropy and lead to a defined value of the spectral density function at zero frequency ${ }^{30}$. Note that the later approach has been implemented on the IDR of a small, partially folded protein: GCN4. Hence, an overall diffusion tensor was defined from relaxation of the folded domain and only internal dynamics where described with the inverse Gaussian approach. Finally, as the conformation energy surface of IDPs can be rightly described as rugged, one could use physical models of motions designed to describe diffusion on a rugged surface, such as fractional diffusion ${ }^{31}$. Unfortunately, this model leads to divergent spectral density function in zero and could only be used in favorable cases where an overall diffusion tensor can be defined, such as GCN4.

\subsection{Comparison with molecular dynamics simulations}

NMR relaxation provides unique information about the amplitude and timescales of motions in proteins in the pico- to nanosecond range with atomic resolution. Yet, this information is not a movie of conformational fluctuations and transitions. NMR is not sufficient to determine a description of the kinetic pathways between conformations. On the other hand, such a description can be achieved by molecular dynamics (MD) simulations, provided MD simulations are in good agreement with experiments. As force fields for molecular dynamics simulations have been developed, historically, to reproduce the properties of folded proteins, agreements between experimental and simulated relaxation rates remain difficult to obtain for IDPs and IDRs.

Yet, MD simulations of the yeast transcription factor GCN4 ${ }^{32}$ have shown semi-quantitative agreement with experiments. This agreement was obtained in part, through a general scaling of order parameters and facilitated by the fact that variations of order parameters in the disordered region are dominated by the natural evolution with the distance to the folded domain. More recently, MD simulations of the disordered protein NTail were performed with state-of-the-art force fields ${ }^{33}$. Relaxation rates were poorly reproduced from the raw trajectories, in particular with simulated transverse relaxation rates higher than experimental rates. Such discrepancies were assigned to the over representation of contacts in the polypeptide chain due to rare encounters over the course of the simulation. Hence a procedure called Average Block Selection Using Relaxation Data (ABSURD) was designed to select ensembles of blocks of the MD trajectory that together reproduced well relaxation rates for all residues. Interestingly, when transverse relaxation rates at a single magnetic field were 
used as a constraint, all simulated rates at all magnetic fields were in better agreement with experimental relaxation rates, demonstrating that the ABSURD procedure corrects for a particular flaw of MD simulations and not for a general failure to simulate properly motions in disordered proteins. An analysis of ABSURD ensembles of sub-trajectories has given a better mechanistic description of the motions that lead to nitrogen-15 relaxation in disordered proteins. This analysis has confirmed the initial interpretation of relaxation datasets, identifying fast librations of the peptide plane, backbone dihedral angle dynamics and rotational diffusion of segments as the main types of motions in the picosecond range, around 1 ns and in the low nanosecond range, respectively ${ }^{34}$.

The weaknesses of molecular dynamics force fields for their application to the simulation of disordered protein were assigned a few years ago mainly to the interaction between water and the polypeptide chain ${ }^{35}$. Recently, Robustelli et al. ${ }^{36}$ introduced a modified force field, with small changes in the van der Walls interaction terms for both water and proteins. This force field described properly conformational properties of both folded and disordered proteins. Accurate unbiased MD simulations of picosecond-nanosecond dynamics of disordered proteins may arise in the near future.

\section{Microsecond-millisecond motions in IDPs and IDRs: binding}

\subsection{Investigating binding of IDPs with titrations.}

Chemical shifts are direct probes of the electronic environment surrounding nuclei. This property can be used to monitor the interaction between a protein and a partner (protein, nucleic acid, or small organic molecules for example) as the later will introduce changes in the electronic cloud of nearby nuclei ${ }^{37}$. A change in the chemical shift of a given nucleus can be due to two distinct effects: participation in the binding for which the chemical shift change is a direct effect of the proximity with a ligand, or wide conformational rearrangement, in which case the partner has long-range effects on the protein and the change in chemical shift of the corresponding nuclei mainly accounts for a new conformational ensemble. The later effects are essentials in the case of allosteric regulation of proteins and can involve folded as well as unfolded proteins or regions ${ }^{38}$.

Unlike any other techniques, NMR guarantees access to ${ }^{37}$ : 
- An atomic resolution of the interaction. This type of information can also be obtained, at least partially, from X-ray crystallography and Cryo-Electron Microscopy;

- The thermodynamics and kinetics of the binding. Isothermal Titration Calorimetry (ITC), Surface Plasmon Resonance (SPR) and Micro-Scale Thermophoresis (MST) are nowadays other reliable instrumentations that can provide this type of information, but it must be pointed out that among these three alternative techniques, only SPR can report on both kinetics and thermodynamics of binding ${ }^{39}$;

All this information can be potentially gathered all at once from a NMR titration experiment, depending on the range of timescales, chemical shift differences and relative populations.

\subsubsection{Atomic resolution of the interaction and model of binding}

NMR is a particularly well-suited tool to study IDPs and IDRs in their complex forms without any prior knowledge of the binding region. This information is absolutely required for X-ray crystallography before any crystallization attempts to design the suitable constructs that first will not prevent crystal formation and second will not lower the quality of the electron density map. Monitoring NMR resonances affected by addition of a partner gives indication on regions that participate in the binding, or that are indirectly affected by the binding and undergo a conformational change. Chemical shifts changes and/or intensity decreases are two clear and easily assessed indicators for binding of IDPs and IDRs (Fig.6). It was for example recently shown that the binding region of Artemis to the DNA binding domain of Ligase-IV has 6 residues contributing significantly to the interaction ${ }^{40}$ that were not present in the electron density map of the complex. It was suggested that these residues are forming fuzzy electrostatic interactions on a large surface area of the Ligase-IV. The residues involved in these poorly-specific electrostatic interactions have a more restrained conformational ensemble compared to the unbound state, but still span a large amount of possible conformations, making the formation of a well-ordered crystal very unlikely. Still, NMR titrations can probe such dynamic contacts.

Moreover, the chemical shift evolution is a clear indication of the number of binding sites using Singular Value Decomposition (SVD) ${ }^{41}$. In this example, ${ }^{1} \mathrm{H}-{ }^{15} \mathrm{~N}$ cross-peaks of the TAZ2 domain of CBP affected by the binding were showing a curved evolution upon addition of the disordered domain of p53. After analysis of the chemical shift evolution during the titration, 
the authors concluded on a two-site binding model for the interaction, with low and high affinity sites for the binding of p53. Another example from the same group shows that NMR titrations can be used to reveal an intermediate bound state stabilized through non-specific intermolecular interactions that evolve to the bound state ${ }^{42}$. In this example, the NMR titration shows an initial fast exchange regime, giving information about the initial non-specific interaction, and evolves to the slow exchange regime for which the intermediate state interconverts with the final bound state that is stabilized by specific intermolecular interactions. These two works show how NMR titrations can be used to study potentially complex binding modes involving sequential events.

Once bound to the folded partner, the high-molecular-weight complex that is formed can dramatically increase transverse relaxation rates of the IDP or IDR at the binding region, leading to line broadening that prevent direct observation of the bound state ${ }^{40}$. Deuteration of IDPs has proven to be efficient to recover cross-peak intensities of regions involved in the interaction with large protein domains ${ }^{43}$. In this study, the spectrum of ${ }^{2}$ D-labeled PNUTS bound to the $38 \mathrm{kDa}$ protein PP1 shows a clear increase in the proton chemical shift dispersion, suggesting a folding-upon-binding transition further confirmed by X-ray crystallography. Interestingly, this example also reveals another aspect of the NMR titration as a way to determine a minimal construct of PNUTS that further led the authors to solve the crystal structure of PP1 bound to the interacting region of the IDR.
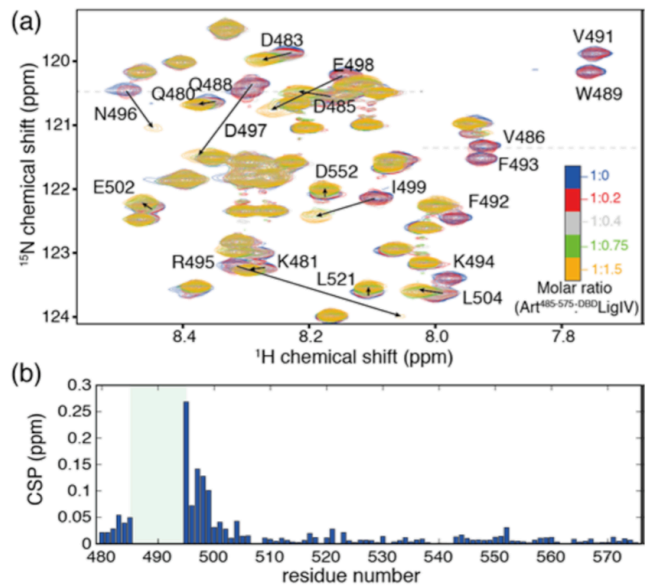

Figure 6. Titration of Artemis (residue 480-675) with the DNA binding domain of Ligase-IV followed by 2D [ $\left[{ }^{1} \mathrm{H}-{ }^{15} \mathrm{~N}\right]-\mathrm{HSQC}$ spectra. Residues essential for the binding (485-495, light green rectangle in (b) and present in the crystal structure) disappear upon binding while residues located on the C-terminus side show significant Chemical Shift Perturbation (CSP). Half of 
these residues are negatively charged and interact with the positively charged surface of Ligase-IV. Reprinted with permission from ${ }^{40}$. Copyright 2017 American Chemical Society.

\subsubsection{Extracting thermodynamics and kinetics information from NMR titration experiments}

As pointed out in a recent review focusing on IDP complexes ${ }^{44}$, NMR titrations can be used to probe a wide range of affinities from picomolar to millimolar. Dissociation constant $K_{d}$ can be obtained in the fast exchange regime $\left(\Delta \omega \ll k_{e x}\right)$ by chemical shift analysis, and in the slow exchange regime $\left(\Delta \omega \gg k_{e x}\right)$ by intensity analysis. However, in all the intermediate situations, neglecting the chemical exchange process leads to systematic error ${ }^{37}$. One way to take into account the effect of the exchange regime on the thermodynamic parameters is to perform lineshape analysis on the recorded spectrum. In protein NMR, and particularly in the case of IDPs and IDRs, two-dimensional lineshape analysis is essential as peak overlaps would prevent the extraction of any reliable information out of one-dimensional spectra. Recently, Waudby et al released an open-source software for the analysis of NMR titrations recorded using different types of experiments (HSQC and HMQC) and isotopic labelling schemes, that considers a selection of two- and three-state binding models (two states, induced fit,...) ${ }^{45}$. This software, called TITAN, relies on the simulation of the two-dimensional spectra, throughout the titration, by using a Liouvillian considering the chemical shift in the free and bound states, the transverse relaxation rates in both states, the dissociation constant(s) and the kinetic constant(s). The NMR experiment is then simulated and the parameters of binding are adjusted to reproduce the recorded spectra (Fig.7). For instance, the Liouvillian used to calculate the density operator in the direct dimension is ${ }^{45}$ :

$$
\begin{aligned}
\frac{d}{d t}\left(\begin{array}{l}
M_{a}(t) \\
M_{b}(t) \\
M_{c}(t)
\end{array}\right)= & -\left[\left(\begin{array}{ccc}
R_{2, a}+i \omega_{a} & 0 & 0 \\
0 & R_{2, b}+i \omega_{b} & 0 \\
0 & 0 & R_{2, c}+i \omega_{c}
\end{array}\right)\right. \\
& \left.-\left(\begin{array}{ccc}
-k_{a b}-k_{a c} & k_{b a} & k_{c a} \\
k_{a b} & -k_{b a}-k_{b c} & k_{c b} \\
k_{a c} & k_{b c} & -k_{c a}-k_{c b}
\end{array}\right)\right]\left(\begin{array}{l}
M_{a}(t) \\
M_{b}(t) \\
M_{c}(t)
\end{array}\right)
\end{aligned}
$$

where $R_{2, i}$ refers to the transverse relaxation rate of state $i, \omega_{i}$ is the frequency of state $i, M_{i}$ is the magnetization of state $\mathrm{i}$ and $\mathrm{k}_{\mathrm{ij}}$ are rate constants from state $\mathrm{i}$ to state $\mathrm{j}$.

Lineshape analysis provides not only information about the thermodynamics of binding (like chemical shift or intensity analysis would provide), but also kinetics and NMR relevant 
information (i.e. transverse relaxation rates) that can further be used to characterize the bound state in terms of dynamics. This relatively large amount of information is obtained from combined analysis of chemical shift, intensity and line broadening evolution.

Titrations analysis requires that both free and bound states (saturated) are observable on the NMR spectra. As discussed earlier, slow tumbling encountered in high-molecular-weight complexes might lead to line-broadening of the peaks of the bound form. In this case, deuteration may solve this issue. Other types of experiments to detect so called "NMR invisible" states can also be used to extract the chemical shifts of the population in the bound state as shown in ${ }^{40}$ and are discussed below.
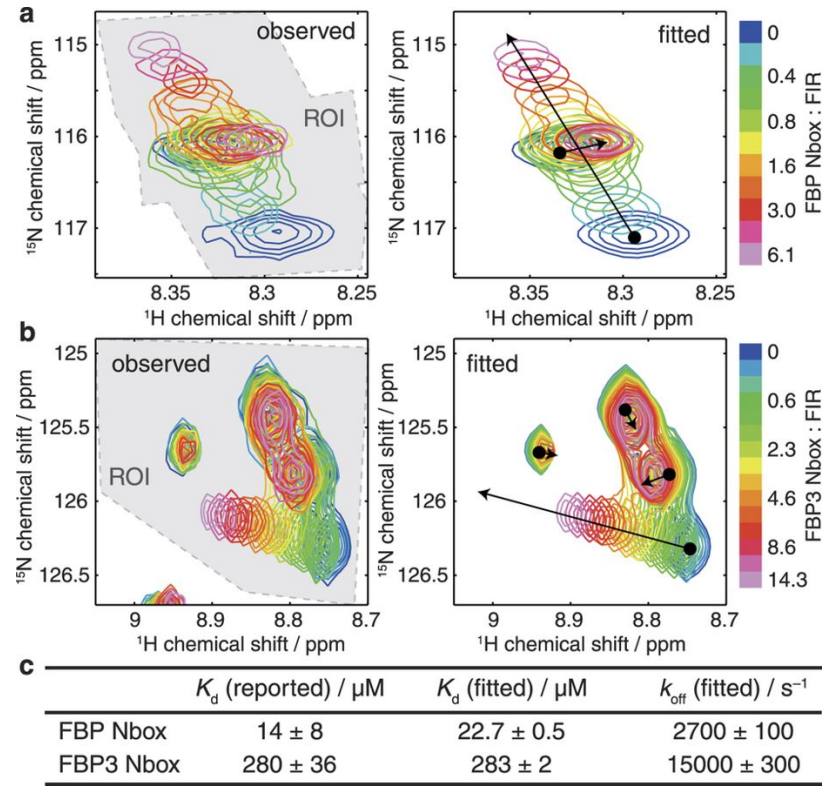

Figure 7. Two-dimensional lineshape analysis. (a) and (b) show two regions of HMQC titrations spectrum of FIR RRM1-RRM2 with FBP box on the left and the corresponding fitted spectrum using TITAN analysis on the right. Extracted thermodynamics values agree with the literature as shown in (c). Reproduced from ${ }^{45}$.

\subsection{Investigations of binding mechanisms with chemical-exchange NMR}

The study of the interaction of an IDP with its partner by NMR is sometimes hindered by the progressive disappearance of NMR signals of key sites from the interacting regions upon addition of the binding partner. In some cases, these disappearing resonances are not recovered even under saturating conditions, where the bound form of the IDP is the only one populated, severely precluding the detailed characterization of the complex using standard NMR techniques. Loss of signal in the course of the titration can be a manifestation of line broadening stemming from conformational exchange on the micro- to millisecond timescale 
between the free and the bound states as well as intermediates along the binding trajectory, or from an increased transverse relaxation rate of the bound state resonance either arising from micro- to millisecond structural dynamics in the complex or simply slow tumbling of the bound parts of the protein in case the complex is of high molecular weight.

NMR techniques designed to study exchange processes in proteins, such as Carr-PurcellMeiboom-Gill (CPMG) relaxation dispersion and chemical exchange saturation transfer (CEST) experiments, allow the characterization of states that are not visible in the spectra, so long as they interconvert with a major, observable state on micro- to millisecond time-scales. Thus, analysis of the NMR-invisible bound form can be achieved by analyzing the relaxation dispersion ${ }^{46}$ or CEST ${ }^{47}$ profiles of resonances from the free protein measured for a sample containing the protein of interest and sub-stoichiometric amounts of the protein partner, so that the bound state is lowly populated.

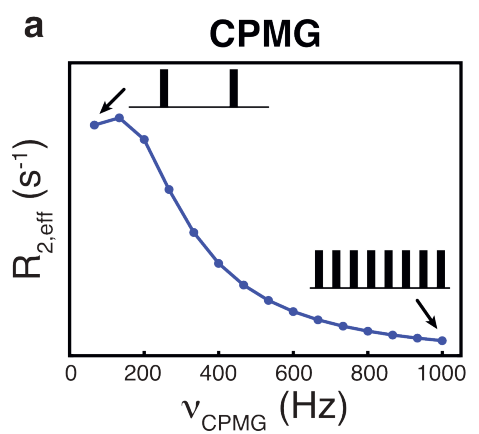

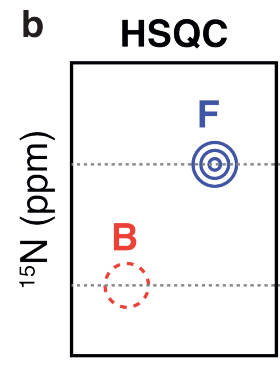

${ }^{1} \mathrm{H}(\mathrm{ppm})$

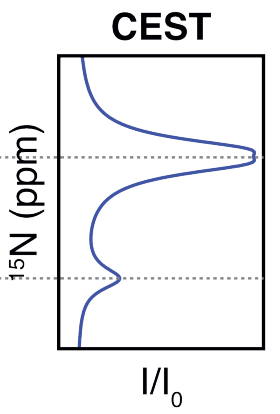

Figure 8. (a) CPMG relaxation dispersion profile. Here, the effective transverse relaxation,

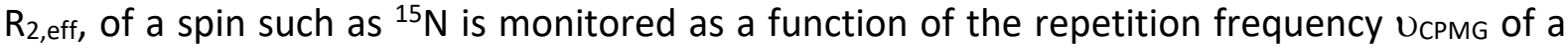
train of refocusing pulses. Higher repetition frequencies attenuate the dephasing of the

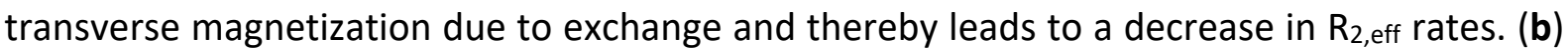
Schematic representation of a small portion of a standard ${ }^{1} \mathrm{H}-{ }^{15} \mathrm{~N}$ two-dimensional correlation spectrum (left), where the peak corresponding to the weakly populated bound state (in red) cannot be observed directly and is only sketched for clarity. The corresponding ${ }^{15} \mathrm{~N}$ CEST profile (right) is obtained by varying the carrier frequency of a weak radio-frequency field. The intensity ratio $\mathrm{I} / \mathrm{I}_{0}$ of the signal of the major state is attenuated when irradiation frequency corresponds to either the free (F) or bound (B) states, thus revealing the position of the 'invisible' B resonance.

The CPMG relaxation dispersion class of experiments ${ }^{48-50}$ builds upon the fact that chemical shift fluctuations arising from stochastic exchange between multiple states induces 
magnetization dephasing, which manifests in increased effective transverse relaxation rates $\left(R_{2, \text { eff }}\right)$. In practice a variable number of chemical shift refocusing pulses is applied during a constant-time period ${ }^{51}$ to modulate the exchange contribution to transverse relaxation rates. Increasing the number of pulses results in a diminution of magnetization dephasing due to exchange and thereby induces a decrease in measured $R_{2, \text { eff }}$ values (Fig. 8a). Subsequent analysis of the variation of $R_{2, \text { eff }}$ as a function of pulsing frequency $\left(v_{C P M G}\right)$, so-called relaxation dispersion (RD) profiles, not only provides information about thermodynamics (binding affinity) and kinetics of the binding reaction, but also site-specific information about the exchanging states in the form of chemical shifts (structural information) and in some favorable cases transverse relaxation rates (dynamic information). Over the last two decades, new pulse sequences along with new spin labeling strategies ${ }^{52}$ have greatly increased the number of nuclear probes that are amenable to study by CPMG RD, including ${ }^{15} \mathrm{~N}^{53,54},{ }^{1} \mathrm{H}^{\mathrm{N}}{ }^{55},{ }^{13} \mathrm{C}^{156},{ }^{13} \mathrm{C}^{\alpha}$ ${ }^{57}$ and ${ }^{1} \mathrm{H}^{\alpha}{ }^{58}$ spin probes.

In CEST experiments ${ }^{59-62}$, a series of spectra are recorded as a function of the position of a weak radio-frequency ( $\mathrm{rf}$ ) field that is applied during a delay where the magnetization of interest is longitudinal. The effect of irradiation is then monitored by plotting the major (observable) state peak intensities (I), normalized to the case where the irradiation delay is absent $\left(I_{0}\right)$, against the rf field offsets. When the irradiation is applied nearby the resonance of a major state, the signal intensity is greatly attenuated due to both spin saturation and "dephasing" induced by rf inhomogeneity. Similarly, when the weak rf field is positioned proximal to a minor ("invisible") state resonance, the perturbation induced by the rf field gets transferred to the major state bulk magnetization via chemical exchange, which again leads to a loss of intensity of the major-state peak. Thus, a typical CEST profile contains a main intensity dip at the frequency of the major state resonance (free form) and a smaller dip at the position corresponding to the minor state resonance ("invisible" bound form) (Fig. 8b). CEST profiles reports on the same exchange parameters as the CPMG relaxation dispersion data. However, the two techniques remain highly complementary when combined together as each depends differently on binding affinity, kinetic model, structure and dynamics of the different states of the binding reaction. While CPMG relaxation dispersion approaches work best for systems with exchange rates that fall roughly into the range $200 \mathrm{~s}^{-1}<k_{\mathrm{ex}}<2000 \mathrm{~s}^{-1}$, CEST is most effective on slower exchange regimes with exchange rates in the range $10 \mathrm{~s}^{-1}<$ 
$k_{\mathrm{ex}}<500 \mathrm{~s}^{-1}$. Importantly, unlike CPMG relaxation dispersion, CEST is relatively insensitive to the presence of ${ }^{13} \mathrm{C}-{ }^{13} \mathrm{C}$ couplings, thereby enabling measurement of NMR-invisible state sidechain ${ }^{13} \mathrm{C}$ chemical shifts in uniformly ${ }^{13} \mathrm{C}$ labeled proteins ${ }^{63,64}$.

In a pioneering study, Sugase, Dyson, and Wright used CPMG relaxation dispersion experiments to decipher the binding mechanism of the disordered phosphorylated kinase inducible activation domain (pKID) of the transcription factor CREB that folds as a pair of helices upon binding onto the KIX domain of the CREB binding protein ${ }^{42}$. NMR titration of pKID with KIX showed that exchange between the free and fully bound states is slow on the chemical shift timescale, but evidence of fast exchange shifts at the early stage of the titration clearly indicated that the binding mechanism is more complex than a simple two-state process. Using ${ }^{15} \mathrm{~N}$ CPMG relaxation dispersion experiments measured at different substoichiometric concentrations of KIX, Wright and co-workers demonstrated that coupled folding and binding of pKID to KIX proceeds through multiple steps with initial diffusional encounter complexes evolving by way of a partially folded intermediate to the final fully folded bound state. Interestingly, in a later study using similar experiments, the same group established that the transcription factor c-Myb interacts with KIX at the same site than pKID but according to different binding mechanism ${ }^{65}$. Here all the data could be analyzed using a simple two-state binding mechanism where no complex intermediates needed to be invoked. Unlike pKID which remain fully disorder in its pre-recognition state, c-Myb exhibits significant helical propensity in its free state reminiscent to the bound state conformation. The authors therefore suggested that the binding mechanisms whereby IDPs interact with their partner strongly depends on their intrinsic secondary structure propensity. In another study ${ }^{66}$, Blackledge and co-workers investigated the binding reaction of the disordered NTAIL domain with its folded, binding partner PX using ${ }^{15} \mathrm{~N},{ }^{13} \mathrm{C}^{\prime}$ and ${ }^{1} \mathrm{H}^{\mathrm{N}}$ CPMG relaxation dispersion experiments recorded at 5 different molar ratios ranging from 2 to $15 \%$ of $\mathrm{PX}$ and 2 magnetic field strengths, 14.1 and $18.7 \mathrm{~T}$. The use of multiple spin probes is interesting as different nuclei can potentially "sense" different processes. For example, ${ }^{13} \mathrm{C}^{\prime}$ resonances are highly dependent on secondary structure and are excellent reporters of folding events while ${ }^{1} \mathrm{H}^{\mathrm{N}}$ and ${ }^{15} \mathrm{~N}$ resonances might also be affected by other molecular processes, such as the formation of specific contacts. In the case of the interaction between NTAIL and PX, the combined analysis of the dispersion data converged towards a binding where NTAIL initially interacts to PX to 
form a non-specific dynamic encounter complex, in which a preformed helical state of free NTAIL is stabilized. This step is followed by the formation of native intermolecular contacts between NTAIL and PX, with NTAIL progressively adopting its final conformation on the surface of PX. In total, these three studies nicely exemplify how CPMG relaxation dispersion approaches can be used to obtain atomic resolution information about complex binding mechanisms involving IDPs and, as such, represent an interesting complement to other kinetic techniques.

As mentioned previously, IDPs can form highly dynamic complexes exhibiting large differences in flexibility along the sequence of the protein, including non- or transiently interacting regions retaining a large amount of disorder as well as segments that fold upon binding or become rigid because tightly bound to the partner. In the NMR spectra, the most flexible parts in rapid exchange between multiple conformers will produce very intense and sharp peaks while folded and tightly bound regions will behave as part of a larger molecule with less favorable relaxation properties, leading to weaker and broader peaks and even full signal disappearance when the complex reaches a certain size ( $\mathrm{MW}>50 \mathrm{kDa}$ ). Bound state resonances can also be broadened out due to conformational dynamics on microsecond-millisecond time scales in the complex. CPMG RD and CEST experiments provide unique opportunities to probe the structure and dynamics of such NMR-challenging complexes as the bound-state resonances do not need to be visible in the spectra to be studied.

One such example studied by Jensen and co-workers is the dynamic complex comprising the 100 amino-acid disordered domain of MKK7, and JNK1, whose interaction is essential to activate the JNK signaling pathway ${ }^{67}$. MKK7 contains three JNK1 docking sites (interaction sites) within its disordered domain but upon addition of JNK1, the resonances from the three docking sites (D1, D2 and D3) of MKK7 progressively disappear providing no insight into the binding mechanism. CPMG relaxation dispersion and CEST experiments were recorded on MKK7 in the presence of $10 \%$ (molar ratio) of JNK1 to obtain structural information about the complex. Both CEST and CPMG experiments reveal exchange on the micro- to millisecond time scale for residues located in and around the docking sites D2 and D3. Interestingly, the analysis of CEST data displays large difference in transverse relaxation rate values between the free and bound states of MKK7 in particular for residues within the D2 docking site. For example, 
a satisfactory fit of the CEST data for T42 requires a $R_{2}$ value larger than $850 \mathrm{~s}^{-1}$ that largely exceeds the expected $R_{2}$ rate for the JNK1-MKK7 complex. This suggests that MKK7 undergoes conformational exchange within the complex and forms a so-called fuzzy complex that is characterized by a number of conformational states. Interestingly, the crystal structure of JNK1 in complex with the second docking site of MKK7 (a 12-residue long peptide) exhibit two different binding conformations of D2 on the surface of JNK1, which supports this view.

More recently, Ferrage and co-workers ${ }^{40}$ have employed CPMG and CEST experiments to characterize the structure and dynamics of a 96-residue long construct from the disordered C-terminal region of the protein Artemis bound to the DNA-binding domain (DBD) of Ligase IV, for which most signals from the interaction site of Artemis could not be detected in the spectra. ${ }^{15} \mathrm{~N}$ CPMG relaxation dispersion experiments on samples of Artemis with increasing amounts of unlabeled Ligase IV (Fig. 9a), complemented with ${ }^{15} \mathrm{~N}$ CEST experiments performed with $10 \%$ molar ratio of Ligase IV (Fig. 9b) were carried out. The combined analysis of the profiles provided accurate ${ }^{15} \mathrm{~N}$ chemical shifts as well as ${ }^{15} \mathrm{~N}$ effective transverse relaxation rates $R_{2}\left({ }^{15} \mathrm{~N}\right)$ for all residues of the bound state of Artemis (Fig. 9f). Intriguingly, $R_{2}\left({ }^{15} \mathrm{~N}\right)$ relaxation rates within the interaction region vary drastically upon binding. While the highest rates were observed at the core of the interaction site (residues 488-494), as expected due to folding and slow molecular tumbling of Ligase IV within the complex, the bound state $R_{2}\left({ }^{15} \mathrm{~N}\right)$ rates for the 495-510 region are lower than in the core of the complex but larger than in the free state. This region spans about twice the persistence length in disordered or unfolded proteins, suggesting a partly restricted conformational space explored on picosecondnanosecond time scales, possibly due to transient interactions with Ligase IV. ${ }^{13} \mathrm{C}$ chemical shifts of Artemis in the bound form were obtained from CEST experiments recorded on ${ }^{13} C^{\prime} 68$ (Fig. 9c), ${ }^{13} \mathrm{C} \alpha{ }^{69}$ (Fig. 9d), and ${ }^{13} \mathrm{C} \beta{ }^{70}$, using a uniformly ${ }^{15} \mathrm{~N},{ }^{13} \mathrm{C}$-labeled sample of Artemis with $10 \%$ molar ratio of unlabelled Ligase IV. These chemical shifts were then used to derive dihedral angles for the backbone and a few side chains of the bound form of Artemis using the program Talos- $\mathrm{N}$, which were subsequently used as structural restraints in the program CNS to produce a structural model of the bound form of Artemis. NMR titrations of the DBD of Ligase IV with Artemis were then performed to collect relevant structural information on the binding surface of Ligase IV. These data were used alongside the structure of free Ligase IV to calculate sets of structures with the docking program HADDOCK. The structural model of 
the complex with lowest energy is remarkably similar to the structures deduced from previous crystallographic studies that used a short 11-amino-acid Artemis peptide, thereby confirming the validity of the approach (Fig. 9e,g).
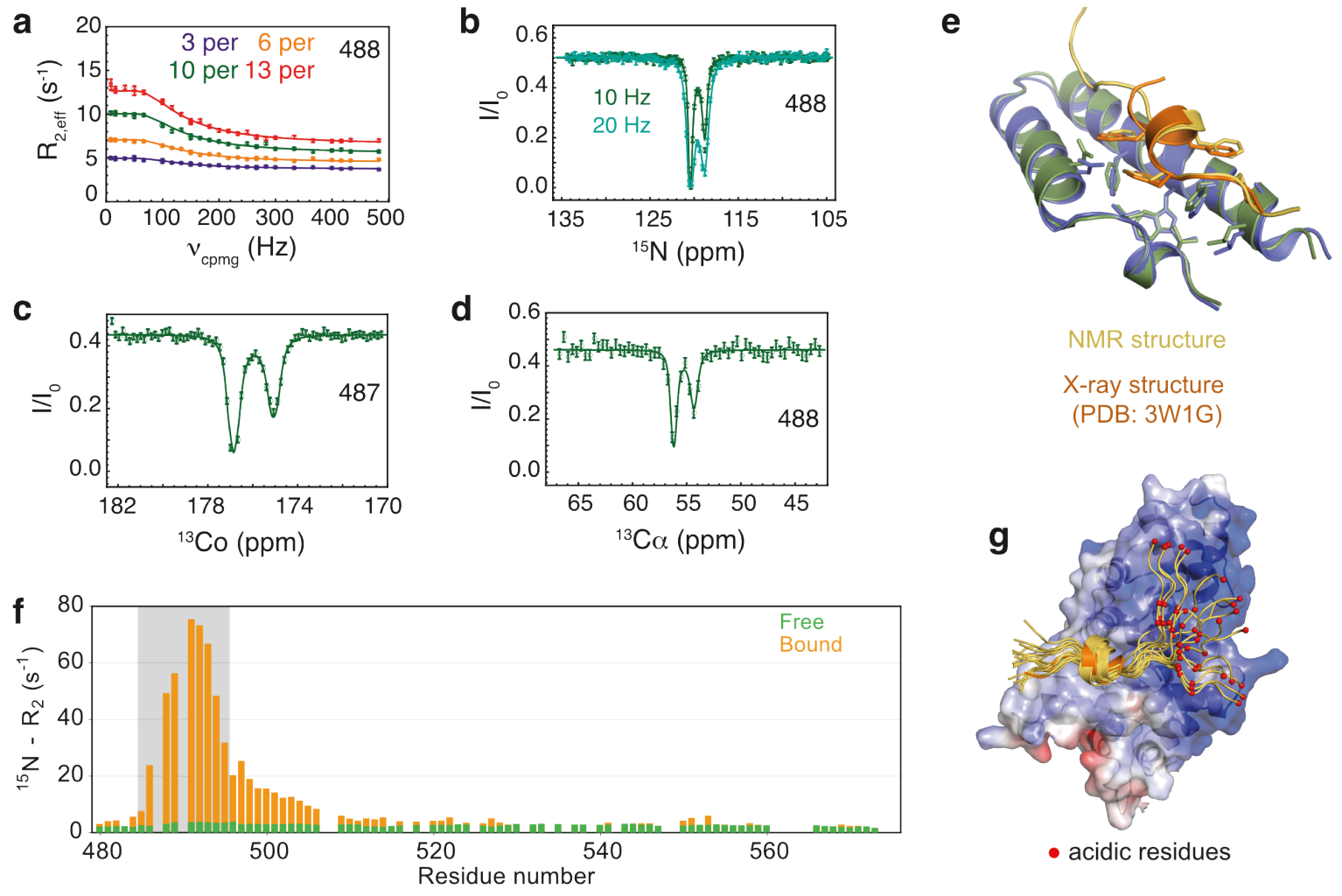

Figure 9. (a) ${ }^{15} \mathrm{~N}$ CPMG dispersion profiles for representative residues of Artemis at the interface with Ligase IV. The concentration of Ligase IV was varied from 0.03 (purple) to 0.06 (orange), 0.10 (green), and 0.13 equiv. (red). (b) ${ }^{15} \mathrm{~N}$ CEST profiles obtained for three representative residues of Artemis. Two different $\mathrm{rf}$ field amplitudes were used: $10.4 \mathrm{~Hz}$ (green) and $20.8 \mathrm{~Hz}$ (light green). Representative ${ }^{13} \mathrm{C}^{\prime}$ (c), and ${ }^{13} \mathrm{C} \alpha$ (d) CEST profiles of nuclei of Artemis at the interface with Ligase IV. Experimental data are shown as small circles. Fitted curves are shown as solid lines in all panels. All CEST experiments were recorded in the presence of 0.1 mol equiv. of unlabeled Ligase IV. (e) Comparison of the contacts at the interface of the complex in the crystal structure (Artemis in orange and Ligase IV in blue, PDB 3 W1G) and in the lowest-energy HADDOCK model of the top cluster (Artemis in yellow and Ligase IV in green). (f) Transverse relaxation rates $R_{2}(15 \mathrm{~N})$ for the free (orange) and bound (green) states of Artemis. (g) The 16 models of HADDOCK top cluster are superimposed onto the crystal structure of the complex as in panel (f). The electrostatic surface potential map of Ligase IV is shown in blue, white, and red to indicate positive, neutral, and negative electrostatic surface potentials, respectively. Residues of Artemis with acidic side-chains (D497, E498, and D501) are represented by small red spheres. Adapted with permission from ${ }^{40}$. Copyright 2017 American Chemical Society. 
In a following study, Jensen and co-workers ${ }^{71}$ applied a very similar approach to decipher the dynamic interaction profile of the intrinsically disordered regulatory domain of the MAPK kinase MKK4 with the MAPK p38 $\alpha$. Here a set of ${ }^{15} \mathrm{~N}$ CEST and CPMG relaxation dispersion experiments recorded on MKK4 at multiple substoichiometric ratios of p38 $\alpha$ was conjointly analyzed to accurately estimate the ${ }^{15} \mathrm{~N}$ transverse relaxation rate values of MKK4 bound to $\mathrm{p} 38 \alpha$. The results show highly heterogeneous $R_{2}$ values of the bound state along the sequence, which is consistent with the presence of multiple dynamic binding modes. The largest values were found at docking site indicating that the motif interacts with p38 $\alpha$ in a stable, persistent manner. Interestingly, lower rates were observed for the KIS domain, suggesting a more dynamic interaction of these residues resembling the one of a fuzzy complex. This study offers a nice illustration of how IDPs can utilize a combination of different interaction modes to bind their partner.

\subsection{Other methods to characterize ps-ns dynamics in complexes of IDPs and IDRs} bound states

In the case of high affinity complexes ( $K_{d}$ on the nanomolar range or tighter), reaching complete saturation is possible from a 1:1 mixture of the two partners. In this case, directly recording relaxation rates on the bound state is possible. For example, Wright and coworkers recently characterized a competition binding event using, among other techniques, the measurement of steady-state $\left\{{ }^{1} \mathrm{H}\right\}{ }^{15} \mathrm{~N}$ NOE for two different IDRs (HIF-1 $\alpha^{776-826}$ and CITED2 ${ }^{216-}$ ${ }^{269}$ ) bound to the TAZ1 ${ }^{340-439}$ domain ${ }^{72}$. Interestingly, these two IDRs bind to the same region of TAZ1 with the same nanomolar affinity. Yet, a 1:1:1 ternary mixture leads exclusively to $100 \%$ of the TAZ1:CITED2 complex. Heteronuclear $\left\{{ }^{1} \mathrm{H}\right\}-{ }^{15} \mathrm{~N}$ NOE allowed a semi-quantitative comparison of ps-ns dynamics of the IDRs in the bound form. The authors found that helix $\alpha_{A}$ in HIF-1 $\alpha$ was still highly dynamic on ps-ns timescales in the bound state, whereas most ps-ns motions were quenched in the analog helix of CITED2. The authors concluded that the helix $\alpha_{A}$ of HIF-1 $\alpha$ was not tightly bound to TAZ1 in the complex, allowing CITED2 helix $\alpha_{A}$ to bind, even if HIF-1 $\alpha$ is already bound (mostly thanks to the interactions of helices $\alpha_{B}$ and $\alpha_{C}$ ) thus forming a transient ternary complex. Further interactions of CITED2 displace HIF-1 $\alpha$ helices $\alpha_{B}$ and $\alpha_{c}$ from TAZ1 to release HIF-1 $\alpha$.

In the case where the complex is not tight but the bound state is still observable on the NMR spectra, measuring NOE and $\mathrm{R}_{1}$ data can be challenging. However, transverse cross-correlated 
cross-relaxation rates (CCCR) are insensitive to chemical exchange while providing an estimate of the spectral density at zero frequency $J(0)$. The use of adiabatic pulses during the relaxation period allows one to improve the sensitivity of the measurements, even in the presence of chemical exchange ${ }^{73}$. This has allowed the characterization of the dynamics of low-populated disordered excited states in the KIX domain of the protein CBP ${ }^{73}$. This method should be readily applicable to the reverse case, where the protein is mostly disordered and the engineered excited state is partly folded in a protein complex.

\subsection{Amide proton exchange: from false positives to insightful information}

Hydrogen/deuterium (H/D) exchange is an indirect way to probe the dynamics and conformational properties of biomolecules in NMR and mass spectrometry, providing information about the solvent accessibility of labile protons. In NMR, it is possible to detect not only $H / D$ exchange in real-time experiments but also to detect exchange of protons with either deuterons or protons from the solvent at the steady state. A very popular experiment used in protein NMR is the Phase-Modulated CLEAN chemical EXchange (CLEANEX-PM) experiment ${ }^{74}$. This experiment was developed for folded proteins, where relaxation is in a regime where the spectral density in zero dominates (slow tumbling regime). This is not the case for IDPs and IDRs, which leads to potential inaccuracies. A series of alternatives have thus been developed.

Nitrogen-15 relaxation rates depend strongly on the dipole-dipole coupling with the bond proton, or deuteron. Thus, longitudinal nitrogen-15 relaxation rates in ${ }^{15} \mathrm{~N}-{ }^{1} \mathrm{H}$ pairs and ${ }^{15} \mathrm{~N}-{ }^{2} \mathrm{H}$ pairs differ significantly. If exchange takes place with a characteristic time comparable to the longitudinal relaxation time, then, $H / D$ exchange rates can be determined from longitudinal relaxation decays measured in a series of samples with different proportions of $\mathrm{H}_{2} \mathrm{O}$ and $\mathrm{D}_{2} \mathrm{O}$ 75. A more straightforward difference between ${ }^{15} \mathrm{~N}-{ }^{1} \mathrm{H}$ and ${ }^{15} \mathrm{~N}-{ }^{2} \mathrm{H}$ pairs is the nitrogen-15 isotropic chemical shift, which differs due to an isotope effect between -0.6 and $-0.7 \mathrm{ppm}{ }^{76}$. The SOLvent EXchange SpectroscopY (SOLEXSY) experiment is performed in a mixture of $\mathrm{H}_{2} \mathrm{O}$ and $\mathrm{D}_{2} \mathrm{O}(50 / 50){ }^{76}$. The evolution of signals arising from ${ }^{15} \mathrm{~N}-{ }^{1} \mathrm{H}$ and ${ }^{15} \mathrm{~N}-{ }^{2} \mathrm{H}$ pairs is monitored in an experiment that selects as an initial state the magnetization of proton-bound nitrogen15 nuclei. Nitrogen-15 signals are monitored in the highly-resolved indirect dimension of $\alpha$ proton detected experiments in ${ }^{13} \mathrm{C} /{ }^{15} \mathrm{~N}$ labeled proteins. Isotope shifts were also recently exploited in a DANTE-Chemical Exchange Saturation Transfer (D-CEST) experiment recorded 
on nitrogen-15 samples ${ }^{77,78}$. In mixture of $\mathrm{H}_{2} \mathrm{O}$ and $\mathrm{D}_{2} \mathrm{O}$ (90/10), D-CEST experiment allow for the characterization of the exchange between ${ }^{15} \mathrm{~N}-{ }^{1} \mathrm{H}$ and ${ }^{15} \mathrm{~N}-{ }^{2} \mathrm{H}$ pairs. This experiment observes exclusively ${ }^{15} \mathrm{~N}-{ }^{1} \mathrm{H}$ pairs but saturation transfer is observed when the nitrogen-15 chemical shift of ${ }^{15} \mathrm{~N}-{ }^{2} \mathrm{H}$ pairs is irradiated. This experiment should allow for the determination of exchange rates up to $100 \mathrm{~s}^{-1}$, with a main limitation being the resolution of the minor dip in CEST profiles. Note that the chemical shift modulation due to the exchange between the hydrogen- and deuterium-bound states may also lead to contributions in the measurement of nitrogen-15 transverse relaxation rates. This has been discussed recently ${ }^{14}$ and may explain many weak dispersions observed in nitrogen-15 CPMG relaxation dispersion experiments in disordered proteins. If such uniform weak dispersions are observed, it is strongly advisable to record the experiment with a different proportion of $\mathrm{D}_{2} \mathrm{O}$ in the sample. If the proportion of the minor species varies accordingly, the effect observed is simply H/D exchange and not conformational exchange.

Finally, in some cases, proton exchange can be too fast to detect either a nitrogen or proton resonance. Such fast exchange is often encountered in physiological conditions: at $37 \stackrel{\circ}{\mathrm{C}}$ and $\mathrm{pH} \sim 7$. In such cases detection on aliphatic protons and triple-resonance methods are necessary, but this is not enough: different physical phenomena have to be used for the detection of exchange. A decade ago, Pelupessy and co-workers introduced the idea that proton exchange could lead to scalar relaxation of the second kind for directly attached nuclei 79,80 . This experiment has been successfully adapted lately for the measurement of amide proton exchange ${ }^{81}$, where exchange rates up to $600 \mathrm{~s}^{-1}$ could be measured at high temperature on a folded protein. In principle exchange rates up to $10^{4} \mathrm{~s}^{-1}$ can be determined. The application to IDPs and IDRs is expected to be straightforward.

\section{References}

(1) Charlier, C.; Cousin, S. F.; Ferrage, F. Protein Dynamics from Nuclear Magnetic Relaxation; Chem. Soc. Rev. 2016, 45, 2410.

(2) Houben, K.; Blanchard, L.; Blackledge, M.; Marion, D. Intrinsic dynamics of the partly unstructured PX domain from the Sendai virus RNA polymerase cofactor P; Biophys. J. 2007, 93, 2830.

(3) Abragam, A. Principles of Nuclear Magnetism; Oxford University Press: Oxford, 1961.

(4) Kowalewski, J. In Nuclear Magnetic Resonance, Vol 40; KamienskaTrela, K., Ed. 2011; Vol. 40, p 205. 
(5) Nicholas, M. P.; Eryilmaz, E.; Ferrage, F.; Cowburn, D.; Ghose, R. Nuclear Spin Relaxation in Isotropic and Anisotropic Media; Prog. Nucl. Magn. Reson. Spectrosc. 2010, in press.

(6) Nowakowski, M.; Saxena, S.; Stanek, J.; Zerko, S.; Kozminski, W. Applications of high dimensionality experiments to biomolecular NMR; Prog. Nucl. Magn. Reson. Spectrosc. 2015, 90-91, 49.

(7) Srb, P.; Nováček, J.; Kadeřávek, P.; Rabatinová, A.; Krásný, L.; Žídková, J.; Bobálová, J.; Sklenář, V.; Žídek, L. Triple resonance $15 \mathrm{~N}$ NMR relaxation experiments for studies of intrinsically disordered proteins; J. Biomol. NMR 2017, 69, 133.

(8) Lawrence, C. W.; Showalter, S. A. Carbon-Detected 15N NMR Spin Relaxation of an Intrinsically Disordered Protein: FCP1 Dynamics Unbound and in Complex with RAP74; The Journal of Physical Chemistry Letters 2012, 3, 1409.

(9) Neuhaus, D.; Williamson, M. P. The Nuclear Overhauser Effect in Structural and Conformational Analysis; 2nd ed.; John Wiley \& Sons: New York, 2000.

(10) Ferrage, F.; Reichel, A.; Battacharya, S.; Cowburn, D.; Ghose, R. On the measurement of $\mathrm{N}-15-\{\mathrm{H}-1\}$ nuclear Overhauser effects. 2. Effects of the saturation scheme and water signal suppression; J. Magn. Reson. 2010, 207, 294.

(11) Lakomek, N.-A.; Ying, J.; Bax, A. Measurement of N-15 relaxation rates in perdeuterated proteins by TROSY-based methods; J. Biomol. NMR 2012, 53, 209.

(12) Khan, S. N.; Charlier, C.; Augustyniak, R.; Salvi, N.; Déjean, V.; Bodenhausen, G.; Lequin, O.; Pelupessy, P.; Ferrage, F. Distribution of pico- and nanosecond motions in disordered proteins from nuclear spin relaxation: a simple array of correlation times; Biophys. J. 2015, 109, 988.

(13) Farrow, N. A.; Zhang, O. W.; Szabo, A.; Torchia, D. A.; Kay, L. E. Spectral Density-Function Mapping Using N-15 Relaxation Data Exclusively; J. Biomol. NMR 1995, 6, 153.

(14) Kumari, P.; Frey, L.; Sobol, A.; Lakomek, N.-A.; Riek, R. $15 \mathrm{~N}$ transverse relaxation measurements for the characterization of $\mu \mathrm{s}-\mathrm{ms}$ dynamics are deteriorated by the deuterium isotope effect on 15N resulting from solvent exchange; J. Biomol. NMR 2018, 72, 125.

(15) Yuwen, T.; Skrynnikov, N. R. Proton-decoupled CPMG: A better experiment for measuring 15N R2 relaxation in disordered proteins; J. Magn. Reson. 2014, 241, 155.

(16) Pelupessy, P.; Espallargas, G. M.; Bodenhausen, G. Symmetrical reconversion: measuring cross-correlation rates with enhanced accuracy; J. Magn. Reson. 2003, 161, 258.

(17) Abyzov, A.; Salvi, N.; Schneider, R.; Maurin, D.; Ruigrok, R. W. H.; Jensen, M. R.; Blackledge, M. Identification of Dynamic Modes in an Intrinsically Disordered Protein Using TemperatureDependent NMR Relaxation; J. Am. Chem. Soc. 2016, 138, 6240.

(18) Marsh, J. A.; Singh, V. K.; Jia, Z. C.; Forman-Kay, J. D. Sensitivity of secondary structure propensities to sequence differences between alpha- and gamma-synuclein: Implications for fibrillation; Protein Sci. 2006, 15, 2795.

(19) Yang, D. W.; Mok, Y. K.; Muhandiram, D. R.; Forman-Kay, J. D.; Kay, L. E. H-1-C-13 dipoledipole cross-correlated spin relaxation as a probe of dynamics in unfolded proteins: Application to the DrkN SH3 domain; J. Am. Chem. Soc. 1999, 121, 3555.

(20) Lipari, G.; Szabo, A. Model-Free Approach to the Interpretation of Nuclear Magnetic Resonance Relaxation In Macromolecules 1. Theory and Range of Validity; J. Am. Chem. Soc. 1982, 104, 4546.

(21) Halle, B. The physical basis of model-free analysis of NMR relaxation data from proteins and complex fluids; J. Chem. Phys. 2009, 131.

(22) Ishima, R.; Nagayama, K. Quasi-spectral-density function-analysis for $n-15$ nuclei in proteins; J. Magn. Reson., Ser B 1995, 108, 73. 
(23) Kaderavek, P.; Zapletal, V.; Rabatinova, A.; Krasny, L.; Sklenar, V.; Zidek, L. Spectral density mapping protocols for analysis of molecular motions in disordered proteins; J. Biomol. NMR 2014, 58, 193.

(24) Lefevre, J.-F.; Dayie, K. T.; Peng, J. W.; Wagner, G. Internal Mobility in the Partially Folded DNA Binding and Dimerization Domains of GAL4: NMR Analysis of the N-H Spectral Density Function; Biochemistry 1996, 35, 2674.

(25) Jensen, M. R.; Bernado, P.; Houben, K.; Blanchard, L.; Marion, D.; Ruigrok, R. W. H.; Blackledge, M. Structural Disorder within Sendai Virus Nucleoprotein and Phosphoprotein: Insight into the Structural Basis of Molecular Recognition; Protein and Peptide Letters 2010, $17,952$.

(26) Smith, A. A.; Ernst, M.; Meier, B. H. Optimized "detectors" for dynamics analysis in solidstate NMR; The Journal of Chemical Physics 2018, 148, 045104.

(27) Buevich, A. V.; Baum, J. Dynamics of unfolded proteins: Incorporation of distributions of correlation times in the model free analysis of NMR relaxation data; J. Am. Chem. Soc. 1999, $121,8671$.

(28) Buevich, A. V.; Shinde, U. P.; Inouye, M.; Baum, J. Backbone dynamics of the natively unfolded pro-peptide of subtilisin by heteronuclear NMR relaxation studies; J. Biomol. NMR 2001, 20, 233.

(29) Cole, K. S.; Cole, R. H. Dispersion and Absorption in Dielectrics I. Alternating Current Characteristics; The Journal of Chemical Physics 1941, 9, 341.

(30) Hsu, A.; Ferrage, F.; Palmer, A. G., III Analysis of NMR Spin Relaxation Data Using an Inverse Gaussian Distribution Function; Biophys. J. 2018, 115, 2301.

(31) Calandrini, V.; Abergel, D.; Kneller, G. R. Fractional protein dynamics seen by nuclear magnetic resonance spectroscopy: Relating molecular dynamics simulation and experiment; J. Chem. Phys. 2010, 133.

(32) Robustelli, P.; Trbovic, N.; Friesner, R. A.; Palmer, A. G. Conformational Dynamics of the Partially Disordered Yeast Transcription Factor GCN4; Journal of Chemical Theory and Computation 2013, 9, 5190.

(33) Salvi, N.; Abyzov, A.; Blackledge, M. Multi-Timescale Dynamics in Intrinsically Disordered Proteins from NMR Relaxation and Molecular Simulation; Journal of Physical Chemistry Letters 2016, 7, 2483.

(34) Salvi, N.; Abyzov, A.; Blackledge, M. Analytical Description of NMR Relaxation Highlights Correlated Dynamics in Intrinsically Disordered Proteins; Angew. Chem. Int. Ed. 2017, 56, 14020.

(35) Best, R. B.; Zheng, W.; Mittal, J. Balanced Protein-Water Interactions Improve Properties of Disordered Proteins and Non-Specific Protein Association; Journal of Chemical Theory and Computation 2014, 10, 5113.

(36) Robustelli, P.; Piana, S.; Shaw, D. E. Developing a molecular dynamics force field for both folded and disordered protein states; Proceedings of the National Academy of Sciences 2018, 115, E4758.

(37) Williamson, M. P. Using chemical shift perturbation to characterise ligand binding; Prog. Nucl. Magn. Reson. Spectrosc. 2013, 73, 1.

(38) Motlagh, H. N.; Wrabl, J. O.; Li, J.; Hilser, V. J. The ensemble nature of allostery; Nature 2014, 508, 331.

(39) Szabo, A.; Stolz, L.; Granzow, R. Surface plasmon resonance and its use in biomolecular interaction analysis (BIA); Curr. Opin. Struct. Biol. 1995, 5, 699. 
(40) Charlier, C.; Bouvignies, G.; Pelupessy, P.; Walrant, A.; Marquant, R.; Kozlov, M.; De loannes, P.; Bolik-Coulon, N.; Sagan, S.; Cortes, P.; Aggarwal, A. K.; Carlier, L.; Ferrage, F. Structure and Dynamics of an Intrinsically Disordered Protein Region That Partially Folds upon Binding by Chemical-Exchange NMR; J. Am. Chem. Soc. 2017, 139, 12219.

(41) Arai, M.; Ferreon, J. C.; Wright, P. E. Quantitative Analysis of Multisite Protein-Ligand Interactions by NMR: Binding of Intrinsically Disordered p53 Transactivation Subdomains with the TAZ2 Domain of CBP; J. Am. Chem. Soc. 2012, 134, 3792.

(42) Sugase, K.; Dyson, H. J.; Wright, P. E. Mechanism of coupled folding and binding of an intrinsically disordered protein; Nature 2007, 447, 1021.

(43) Choy, M. S.; Hieke, M.; Kumar, G. S.; Lewis, G. R.; Gonzalez-DeWhitt, K. R.; Kessler, R. P.; Stein, B. J.; Hessenberger, M.; Nairn, A. C.; Peti, W.; Page, R. Understanding the antagonism of retinoblastoma protein dephosphorylation by PNUTS provides insights into the PP1 regulatory code; Proceedings of the National Academy of Sciences 2014, 201317395.

(44) Schneider, R.; Blackledge, M.; Jensen, M. R. Elucidating binding mechanisms and dynamics of intrinsically disordered protein complexes using NMR spectroscopy; Curr. Opin. Struct. Biol. 2019, 54, 10.

(45) Waudby, C. A.; Ramos, A.; Cabrita, L. D.; Christodoulou, J. Two-Dimensional NMR Lineshape Analysis; Scientific Reports 2016, 6, 24826.

(46) Sugase, K.; Lansing, J. C.; Dyson, H. J.; Wright, P. E. Tailoring Relaxation Dispersion Experiments for Fast-Associating Protein Complexes; J. Am. Chem. Soc. 2007, 129, 13406.

(47) Sekhar, A.; Rumfeldt, J. A. O.; Broom, H. R.; Doyle, C. M.; Bouvignies, G.; Meiering, E. M.; Kay, L. E. Thermal fluctuations of immature SOD1 lead to separate folding and misfolding pathways; Elife 2015, 4.

(48) Carr, H. Y.; Purcell, E. M. Effects of Diffusion on Free Precession in Nuclear Magnetic Resonance Experiments; Physical Review 1954, 94, 630.

(49) Meiboom, S.; Gill, D. Modified Spin-Echo Method for Measuring Nuclear Relaxation Times; Rev. Sci. Instrum. 1958, 29, 688.

(50) Palmer III, A. G.; Kroenke, C. D.; Loria, J. P. Nuclear magnetic resonance methods for quantifying microsecond-to-millisecond motions in biological macromolecules; Meth. Enzymol. 2001, 339, 204.

(51) Mulder, F. A. A.; Skrynnikov, N. R.; Hon, B.; Dahlquist, F. W.; Kay, L. E. Measurement of slow (mu s-ms) time scale dynamics in protein side chains by $\mathrm{N}-15$ relaxation dispersion NMR spectroscopy: Application to Asn and Gln residues in a cavity mutant of T4 lysozyme; J. Am. Chem. Soc. 2001, 123, 967.

(52) Lundström, P.; Vallurupalli, P.; Hansen, D. F.; Kay, L. E. Isotope labeling methods for studies of excited protein states by relaxation dispersion NMR spectroscopy; Nature Protocols 2009, 4, 1641.

(53) Loria, J. P.; Rance, M.; Palmer, A. G. A relaxation-compensated Carr-Purcell-Meiboom-Gill sequence for characterizing chemical exchange by NMR spectroscopy; J. Am. Chem. Soc. 1999, 121, 2331.

(54) Hansen, D. F.; Vallurupalli, P.; Kay, L. E. An improved N-15 relaxation dispersion experiment for the measurement of millisecond time-scale dynamics in proteins; J. Phys. Chem. B 2008, 112, 5898.

(55) Ishima, R.; Torchia, D. A. Extending the range of amide proton relaxation dispersion experiments in proteins using a constant-time relaxation-compensated CPMG approach; J. Biomol. NMR 2003, 25, 243. 
(56) Ishima, R.; Baber, J.; Louis, J. M.; Torchia, D. A. Carbonyl carbon transverse relaxation dispersion measurements and $\mathrm{ms}$-mu s timescale motion in a protein hydrogen bond network; J. Biomol. NMR 2004, 29, 187.

(57) Hansen, D. F.; Vallurupalli, P.; Lundstrom, P.; Neudecker, P.; Kay, L. E. Probing chemical shifts of invisible states of proteins with relaxation dispersion NMR spectroscopy: How well can we do?; J. Am. Chem. Soc. 2008, 130, 2667.

(58) Vallurupalli, P.; Hansen, D. F.; Lundstrom, P.; Kay, L. E. CPMG relaxation dispersion NMR experiments measuring glycine $\mathrm{H}-1$ (alpha) and $\mathrm{C}-13$ (alpha) chemical shifts in the 'invisible' excited states of proteins; J. Biomol. NMR 2009, 45, 45.

(59) Forsen, S.; Hoffman, R. A. Study of moderately rapid chemical exchange reactions by means of nuclear magnetic double resonance; J. Chem. Phys. 1963, 39, 2892.

(60) Fawzi, N. L.; Ying, J.; Ghirlando, R.; Torchia, D. A.; Clore, G. M. Atomic-resolution dynamics on the surface of amyloid-beta protofibrils probed by solution NMR; Nature 2011, 480, 268.

(61) Vallurupalli, P.; Bouvignies, G.; Kay, L. E. Studying "Invisible" Excited Protein States in Slow Exchange with a Major State Conformation; J. Am. Chem. Soc. 2012, 134, 8148.

(62) Vallurupalli, P.; Sekhar, A.; Yuwen, T.; Kay, L. E. Probing conformational dynamics in biomolecules via chemical exchange saturation transfer: a primer; J. Biomol. NMR 2017, 67, 243.

(63) Bouvignies, G.; Vallurupalli, P.; Kay, L. E. Visualizing Side Chains of Invisible Protein Conformers by Solution NMR; J. Mol. Biol. 2014, 426, 763.

(64) Vallurupalli, P.; Bouvignies, G.; Kay, L. E. A Computational Study of the Effects of 13C-13C Scalar Couplings on 13C CEST NMR Spectra: Towards Studies on a Uniformly 13C-Labeled Protein; ChemBioChem 2013, 14, 1709.

(65) Arai, M.; Sugase, K.; Dyson, H. J.; Wright, P. E. Conformational propensities of intrinsically disordered proteins influence the mechanism of binding and folding; Proceedings of the National Academy of Sciences 2015, 112, 9614.

(66) Schneider, R.; Maurin, D.; Communie, G.; Kragelj, J.; Hansen, D. F.; Ruigrok, R. W. H.; Jensen, M. R.; Blackledge, M. Visualizing the Molecular Recognition Trajectory of an Intrinsically Disordered Protein Using Multinuclear Relaxation Dispersion NMR; J. Am. Chem. Soc. 2015, 137, 1220.

(67) Kragelj, J.; Palencia, A.; Nanao, M. H.; Maurin, D.; Bouvignies, G.; Blackledge, M.; Jensen, M. R. Structure and dynamics of the MKK7-JNK signaling complex; Proc. Natl. Acad. Sci. U. S. A. 2015, 112, 3409.

(68) Vallurupalli, P.; Kay, L. E. Probing Slow Chemical Exchange at Carbonyl Sites in Proteins by Chemical Exchange Saturation Transfer NMR Spectroscopy; Angew. Chem. 2013, 125, 4250.

(69) Motackova, V.; Novacek, J.; Zawadzka-Kazimierczuk, A.; Kazimierczuk, K.; Zidek, L.; Anderova, H. S.; Krasny, L.; Kozminski, W.; Sklenar, V. Strategy for complete NMR assignment of disordered proteins with highly repetitive sequences based on resolution-enhanced $5 \mathrm{D}$ experiments; J. Biomol. NMR 2010, 48, 169.

(70) Long, D.; Sekhar, A.; Kay, L. E. Triple resonance-based $13 \mathrm{C} \alpha$ and $13 \mathrm{C} \beta$ CEST experiments for studies of ms timescale dynamics in proteins; J. Biomol. NMR 2014, 60, 203.

(71) Delaforge, E.; Kragelj, J.; Tengo, L.; Palencia, A.; Milles, S.; Bouvignies, G.; Salvi, N.; Blackledge, M.; Jensen, M. R. Deciphering the Dynamic Interaction Profile of an Intrinsically Disordered Protein by NMR Exchange Spectroscopy; J. Am. Chem. Soc. 2018, 140, 1148.

(72) Berlow, R. B.; Dyson, H. J.; Wright, P. E. Hypersensitive termination of the hypoxic response by a disordered protein switch; Nature 2017, 543, 447. 
(73) Kadeřávek, P.; Grutsch, S.; Salvi, N.; Tollinger, M.; Žídek, L.; Bodenhausen, G.; Ferrage, F. Cross-correlated relaxation measurements under adiabatic sweeps: determination of local order in proteins; J. Biomol. NMR 2015, 63, 353.

(74) Hwang, T. L.; van Zijl, P. C. M.; Mori, S. Accurate quantitation of water-amide proton exchange rates using the Phase-Modulated CLEAN chemical EXchange (CLEANEX-PM) approach with a Fast-HSQC (FHSQC) detection scheme; J. Biomol. NMR 1998, 11, 221.

(75) Lopez, J.; Ahuja, P.; Landrieu, I.; Cantrelle, F.-X.; Huvent, I.; Lippens, G. H/D exchange of a $15 \mathrm{~N}$ labelled Tau fragment as measured by a simple Relax-EXSY experiment; J. Magn. Reson. 2014, 249, 32.

(76) Chevelkov, V.; Xue, Y.; Krishna Rao, D.; Forman-Kay, J. D.; Skrynnikov, N. R. 15NH/DSOLEXSY experiment for accurate measurement of amide solvent exchange rates: application to denatured drkN SH3; J. Biomol. NMR 2010, 46, 227.

(77) Yuwen, T.; Kay, L. E.; Bouvignies, G. Dramatic Decrease in CEST Measurement Times Using Multi-Site Excitation; ChemPhysChem 2018, 19, 1707.

(78) Yuwen, T.; Bah, A.; Brady, J. P.; Ferrage, F.; Bouvignies, G.; Kay, L. E. Measuring Solvent Hydrogen Exchange Rates by Multi-Frequency Excitation 15N CEST; The Journal of Physical Chemistry B 2018.

(79) Kateb, F.; Abergel, D.; Blouquit, Y.; Duchambon, P.; Craescu, C. T.; Bodenhausen, G. Slow Backbone Dynamics of the C-Terminal Fragment of Human Centrin 2 in Complex with a Target Peptide Probed by Cross-Correlated Relaxation in Multiple-Quantum NMR Spectroscopy; Biochemistry 2006, 45, 15011.

(80) Segawa, T.; Kateb, F.; Duma, L.; Bodenhausen, P.; Pelupessy, P. Exchange Rate Constants of Invisible Protons in Proteins Determined by NMR Spectroscopy ChemBioChem 2008, 9, 537. (81) Dass, R.; Corlianò, E.; Mulder, F. A. A. Measurement of Very Fast Exchange Rates of Individual Amide Protons in Proteins by NMR Spectroscopy; ChemPhysChem 2018, 0 . 